\title{
BMJ Open Self-care interventions in stroke survivor-caregiver dyads: a protocol for systematic review and meta-analysis
}

\author{
Wenna Wang, ${ }^{1}$ Beilei Lin, ${ }^{2}$ Yongxia Mei, ${ }^{2}$ Zhenxiang Zhang (D) ${ }^{2}$ Bing Zhou ${ }^{3}$
}

To cite: Wang W, Lin B, Mei Y, et al. Self-care interventions in stroke survivor-caregiver dyads a protocol for systematic review and meta-analysis. BMJ Open 2021;11:e051860. doi:10.1136/ bmjopen-2021-051860

- Prepublication history and additional supplemental material for this paper are available online. To view these files, please visit the journal online (http://dx.doi.org/10.1136/ bmjopen-2021-051860)

Received 30 March 2021 Accepted 22 November 2021

Check for updates

(c) Author(s) (or their employer(s)) 2021. Re-use permitted under CC BY-NC. No commercial re-use. See rights and permissions. Published by BMJ.

${ }^{1}$ Academy of Medical Sciences, Zhengzhou University,

Zhengzhou, China

${ }^{2}$ School of Nursing and Health, Zhengzhou University,

Zhengzhou, China

${ }^{3}$ School of Information

Engineering, Zhengzhou University, Zhengzhou, China

Correspondence to

Dr Zhenxiang Zhang;

zhangzx6666@zzu.edu.cn

\section{ABSTRACT}

Introduction Stroke is known as one of the leading causes of mortality and disability worldwide. Self-care plays a significant role in improving the quality of life, self-efficacy and many other outcomes of stroke survivors. However, it is a dyadic phenomenon where patient selfcare and the caregiver contribution to self-care are interrelated in terms of predictors and outcomes. Currently, there is still no systematic assessment conducted to examine the overall effectiveness of self-care interventions carried out in stroke survivor-caregiver dyads and explore the effect on stroke survivor and/or caregiver outcomes. Methods We plan to conduct a systematic review and meta-analysis of the evidence regarding the self-care interventions carried out in stroke survivor-caregiver dyads. We will undertake a systematic search of multiple databases including PubMed, Web of Science, CINAHL, PsycINFO, EMBASE, Cochrane Central Register of Controlled Trials and four Chinese databases (CNKI, CBM, WANFANG and VIP) from inception to July 2021 for the purpose of collecting the relevant articles. The eligible studies are defined as those original researches, written in English or Chinese, on self-care interventions in stroke survivor-caregiver dyad samples. Two independent researchers will be deployed to identify the eligible trials according to the selection criteria and extract the relevant data. The Preferred Reporting Items for Systematic Review and Meta-Analysis Protocols checklist has been used for this protocol. We will use the Cochrane Risk for Bias tool to assess the risk of bias for randomised controlled trials. Ethics and dissemination In our review, any identifiable patient data will be excluded, which removes the need for ethical approval and participant consent. The final results of our study will be published in an open-access peerreviewed journal, and abstract will be presented at suitable national/international conferences.

PROSPERO registration number CRD42021239824.

\section{INTRODUCTION}

Stroke is known as one of the leading causes of mortality and disability that incurs significant societal cost and burden. ${ }^{1}$ According to the Global Burden of Disease Study, there were about 80 million stroke survivors worldwide in 2016, apart from 13.7 million new stroke survivors each year. ${ }^{2}$ According to a recent systematic review (SR), the cumulative recurrence rate reached $7.7 \%$ and $9.5 \% 3$ months

\section{Strengths and limitations of this study}

In contrast to other published reviews, our study will be presented as the first systematic review conducted to compile the evidence on self-care interventions carried out in stroke survivor-caregiver dyads.

- In our study, a search will be conducted for those articles published both in English and Chinese. Besides, our team has systematically added Chinese research database and provided this large amount of data to non-Chinese readers.

- The results of our study will provide evidence suggesting the effect of dyadic self-care interventions and identify the most promising elements of intervention for guiding the clinical practice of nursing, so as to improve health outcomes for stroke survivors and caregivers.

- A potential limitation of our study is that this is an understudied research area and there would be few studies identified in this review. As a result, heterogeneity will arise in the process of meta-analysis.

and 6 months after stroke, respectively, with $13.2 \%$ and $34.8 \%$ of all stroke inpatients getting hospitalised due to the recurrence of stroke. ${ }^{3}$ The poor functional, cognitive and psychological outcomes manifested after stroke, such as haemiplegia, aphasia and depression, have negative effects on a large proportion of long-term stroke survivors. ${ }^{4}$ In some studies, it has been demonstrated that the good self-care after stroke can contribute to the recovery of various physiological functions for patients, thus improving the quality of their life. In the meantime, stroke recurrence is prevented and the readmission rate is reduced. ${ }^{56}$ In 8760 hours throughout a year, patients spend merely $0.001 \%$ of their time with medical staff, which means that all other activities related to health maintenance, monitoring and management are carried out either by individuals or by patients and their families as the self-care activities performed outside the clinical or hospital environment. ${ }^{5}$ The study of Haley showed that stroke survivor and caregiver reports of engagement were 
closely correlated with each other $(\mathrm{r}=0.89) .{ }^{7}$ In practice, self-care is often cocare for stroke survivors. That is to say, patients often practice stroke self-care together with their informal caregivers, especially those family members who exert significant influence on the self-care process. ${ }^{89}$ Additionally, there have been more and more theoretical and practical studies indicating that stroke can be viewed as a dyad event, which can affect the physical, psychological and social functions of patients and their caregivers. ${ }^{10}$

Self-care is defined as a naturalistic decision-making process aimed to address both the prevention and management of chronic illness. ${ }^{5}$ According to the MiddleRange Theory of Self-Care of Chronic Illness, self-care is interpreted as a process of maintaining health. ${ }^{11}$ Besides, it can be regarded as an overarching construct based on the three critical concepts including self-care maintenance, self-care monitoring and self-care management. As for self-care maintenance, it refers to those behaviours performed to improve well-being, maintain health or ensure physical and emotional stability. Self-care monitoring is defined as a process of routine, vigilant body monitoring, surveillance or 'body listening'. Self-care management requires an evaluation of the changes in various physical and emotional signs and symptoms to determine whether it is necessary to take action in response. The performance of sufficient self-care is supposed to encompass all three behaviours because these three concepts are closely related to each other. ${ }^{12}$ Self-care represents a fundamental element of the treatment received by those patients with chronic conditions and is a major focus of many interventions. Self-care interventions can play a role in increasing choice, improving accessibility and affordability as well as presenting opportunities for individuals to make informed decisions on their health and healthcare. ${ }^{13}$ Despite a series of SRs suggesting that the self-care interventions/programmes, which target only patients improved the quality of life (QoL), self-efficacy, coping ability as well as activities of daily living (ADL) and social participation for patients with stroke, which makes them different from inactive or active (usual care) control interventions, there remain some limitations on these self-care interventions, for example, the self-management compliance of stroke survivors was poor, some self-care skills could not be developed independently by them, and the contributions of stroke caregivers could not be ignored in the self-care of stroke. ${ }^{14-16}$

Typically, two individuals (such as husband and wife) maintaining a sociologically important relationship are defined as a dyad. ${ }^{17}$ According to the theory of dyadic illness management, the patients suffering chronic diseases and their caregivers take joint participation in disease management, which involves decision-making, emotional communication support, the management of changes in physical function and general health behaviours. The ultimate goal of doing this is to promote the dual health of patients and their caregivers. ${ }^{18}$ In previous studies, it has been demonstrated that the health outcomes of stroke survivors and caregivers affected each other and was greatly impacted by the physical function changes of the survivor. Therefore, the dyadic approaches to stroke care that recognise the interdependence of dyads are required. ${ }^{19} 20$ The self-care interventions carried out in stroke survivor-caregiver dyads are delivered to both a patient and his/her informal caregiver with the expectations that both dyad members will be actively engaged in the stroke self-care of patients. Given the significance of dyadic perspectives in stroke self-care, it is essential to summarise the self-care interventions carried out in survivor-caregiver dyads and understand how the dyadic self-care interventions developed and implemented can improve the health outcomes of stroke survivor-caregiver dyads. Therefore, the aim of our study is to determine what self-care interventions implemented in stroke survivor-caregiver dyads can contribute to improving health-related outcomes for stroke survivors and caregivers and analyse the overall effectiveness of selfcare interventions conducted in stroke survivor-caregiver dyads through meta-analysis (MA), thus identifying the most effective intervention in improving health-related outcomes for both stroke survivors and caregivers.

\section{OBJECTIVES}

The purpose of our review is to integrate the scientific evidence on dyadic self-care interventions for enhancing the health-related outcomes for stroke survivors and caregivers, which entails an understanding of the influencing factors in the success of interventions with healthrelated outcomes for stroke survivors and caregivers. Therefore, attempt will be made to answer the following research questions. First, what is the overall effectiveness of self-care interventions carried out in stroke survivorcaregiver dyads with health-related outcomes for stroke survivors, covering the QoL, self-efficacy, coping ability, emotional functioning, stroke self-care behaviours, social participation and ADL, and for caregivers, covering the QoL, self-efficacy, burden, coping ability and emotional functioning. Second, how do the effects of dyadic selfcare interventions on health-related outcomes vary according to the characteristics of study design and the risk of bias? Finally, how do the effects of dyadic self-care interventions on health-related outcomes vary depending on the characteristics of intervention, such as the type of patient populations, intervention content, the combination of intervention components, intervention dose and the composition of interventionists?

\section{METHODOLOGY}

Our study will use a SR and MA, which will consider both randomised controlled trials and non-randomised trials (prospective and retrospective observational studies). The Preferred Reporting Items for Systematic Review and Meta-Analysis Protocols (PRISMA-P) statement has been used in the preparation of our protocol..$^{21}$ And we will 
follow the PRISMA guidelines and standard methods for conducting an SR and MA, ${ }^{22}$ while the random effect MA will be conducted to thoroughly evaluate the research results. In addition, exploratory moderator analysis will be carried out to account for heterogeneity.

\section{Selection criteria}

Our study aims to identify the studies published from inception to July 2021 of stroke survivor-caregiver dyads. According to the latest science papers on stroke self-care in survivor-caregiver dyads, the intervention included in our review is supposed to involve at least one of the following elements: (a) self-monitoring coping with the condition, (b) performing regular medication, exercising and other self-sustaining behaviours, (c) goal setting, (d) decision-making, (e) problemsolving, (f) an alternative method designed to facilitate behaviour change and improvements in physical and psychological functioning. ${ }^{23-28}$ The inclusion criteria applied to all papers are detailed as follows. The participants are aged 18 or over; dyad consists of a patient with stroke and at least one informal caregiver; both dyad members should be targeted for the intervention, present at the intervention outcomes (primary or secondary) from both OR either one of the participants measured; randomised controlled trials are conducted to include cross-over studies and cluster studies. What to be excluded from consideration includes meta-analyses and SRs, duplicative papers, case reports, opinion pieces, editorials and letters written to the editor. Besides, quasi-experiment trials or other intervention trials and the interventions that provided education or exercise only to participants will be excluded. In addition, we will exclude the studies of patients with cognitive impairment or institutionalised individuals because their stroke self-care is commonly managed by medical professionals. Additionally, we will include the studies under review, which may provide additional components about self-care interventions. Our study will include only those papers published in English or Chinese and conducted among human subjects only. However, we will also include non-English/Chinese studies if an Englishlanguage version of the abstract containing sufficient data is available for the calculation of effect size (ES).

\section{Data sources and search strategy}

We will conduct a systematic search of electronic database, including PubMed, Web of Science, CINAHL, PsycINFO, EMBASE, Cochrane Central Register of Controlled Trials and four Chinese databases (National Knowledge Infrastructure (CNKI), Chinese Biomedical Literatures database (CBM), Wanfang Digital Periodicals (WANFANG) and Chinese Science and Technology Periodicals (VIP) database) ) from inception to July 2021, for the collection of relevant articles. To apply a variety of different search strategies is conducive to eliminating the risk of bias in MA results. ${ }^{29}{ }^{30}$ To avoid the prospect of missing the eligible literature, we will conduct ancestry searches using the reference lists of eligible studies and conduct a review of the articles published over the past decade. Besides, we will search those unpublished studies and contact the experts in the field of stroke and self-care to identify any additional trials. The search strategy adopted for PubMed has been reviewed by the experts in different fields of stroke care, as listed in table 1. See online supplemental file 1 for full search strategies for all databases.

\section{Methodological quality assessment}

We will use the Cochrane Collaboration's Risk for Bias tool to evaluate the risk of bias and the quality of the studies in the form of randomised controlled trials, ${ }^{31}$ as shown in table 2. Also, this tool allows each domain of potential bias to be classified either as 'low risk', 'high risk' or 'unclear risk'. ${ }^{32}$ Apart from that, consideration will be given to methodological quality as an empirical question, the coding data about aspects of study quality and the potential risks of bias. Then, the data will be subjected to moderator analyses for establishing whether the potential risks of bias are significantly associated with the ES of the study. ${ }^{33}$ Two researchers will carry out this process independently, and any disagreement will be resolved through discussion and consultation with the third researcher, if necessary. The selection process will be presented as a PRISMA flow diagram (figure 1).

\section{Data collection and extraction}

We will use an EndNote V.X9 database to manage all citations from searches, so as to facilitate the screening of titles and abstracts. The two well-trained researchers (WW and BL) will screen a random sample of 50 studies in line with the study selection criteria to assess the consistency of literature selection. Once the interrater agreement measured by Kappa falls below 0.8, secondary training will be provided. ${ }^{34}$ The two researchers will screen all the initial studies independently if the agreement has been reached. Otherwise, the third researcher (YM) will intervene to resolve the disagreement between the first two reviewers. In the screening performed by the two welltrained researchers, titles and abstracts will be examined to evaluate whether the study involves an intervention purposed to improve an aspect of stroke self-care, with any possibly eligible citations marked for the retrieval of the entire paper. Any discrepancies will be discussed whenever necessary until the final studies are all identified.

These two researchers (WW and BL) will carry out data extraction independently. First, the data will be extracted from a random sample of 10 studies to verify the consistence of data extraction between WW and BL. If the level of agreement falls below 0.8 , the causes will be explored and adjustment will be made. Data extraction will cover the following information: (a) study characteristics, including the first author's name, publication year, country, title, studies design and settings, (b) participants, including the type of dyads, sample sizes, inclusion and exclusion criteria, sex ratio and average age, (c) methodological quality of trials, including the details on random process, blinding, dropout, reporting and 
Table 1 PubMed search strategy

\begin{tabular}{|c|c|}
\hline PubMed & \\
\hline 1 & Search ("stroke"(MeSH Terms)) \\
\hline 2 & $\begin{array}{l}\text { Search ("cva"(Title/Abstract)OR "stroke*"(Title/Abstract)OR "poststroke*"(Title/Abstract)OR "post stroke*"(Title/ } \\
\text { Abstract)OR "transient ischemic attack“"(Title/Abstract)OR "TIA"(Title/Abstract)OR "ministroke*"(Title/Abstract)OR } \\
\text { "SAH"(Title/Abstract)) }\end{array}$ \\
\hline 3 & $\begin{array}{l}\text { Search ("cerebrovascular disorders"(Title/Abstract)OR "brain ischemia"(Title/Abstract)OR "brain infarction"(Title/ } \\
\text { Abstract)OR "intracranial embolism"(Title/Abstract)OR "thrombosis"(Title/Abstract)OR "intracranial } \\
\text { hemorrhages"(Title/Abstract)) }\end{array}$ \\
\hline 4 & \#1 OR \#2 OR \#3 \\
\hline 5 & 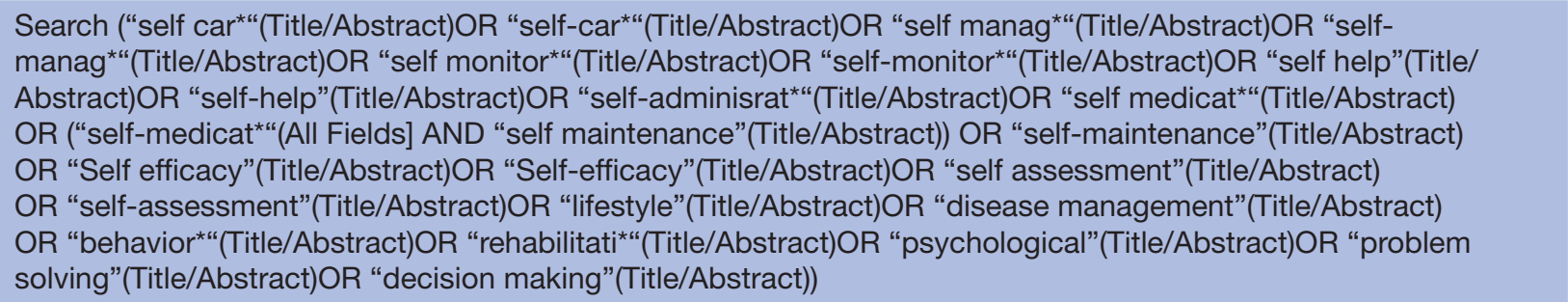 \\
\hline 6 & $\begin{array}{l}\text { Search ((((“Sexual Partners”(MeSH Terms)) OR “spouses”(MeSH Terms])OR “family”(MeSH Terms)) OR } \\
\text { "caregivers”(MeSH Terms)) }\end{array}$ \\
\hline 7 & $\begin{array}{l}\text { Search ("informal care"(Title/Abstract)OR "caregiver*"(Title/Abstract)OR "spous*"(Title/Abstract)OR "husband"(Title/ } \\
\text { Abstract)OR "wife"(Title/Abstract)OR "family"(Title/Abstract)OR "families"(Title/Abstract)OR "son"(Title/Abstract)OR } \\
\text { "daughter"(Title/Abstract)OR "parents"(Title/Abstract)OR "partner*“(Title/Abstract)OR "couple*"(Title/Abstract)OR } \\
\text { "carer"(Title/Abstract)OR "carers"(Title/Abstract)OR "dyad*"(Title/Abstract)) }\end{array}$ \\
\hline 8 & \#6 OR \#7 \\
\hline 9 & \#4 AND \#5 AND \#8 \\
\hline
\end{tabular}

others, (d) intervention information, including intervention contexts, types, frequency, intensity, duration and other intervention components, (e) outcomes information, including the indicators of primary outcomes or secondary outcomes, measurement tools, the related statistics such as mean \pm SD, p value and 95\% CI and intention-to-treat analysis. We will use a designed dataencoding form to code the information extracted from the included studies. When mean and SD, postintervention data and others are not directly available in the study, attempt will be made to obtain them through calculation according to the Cochrane handbook and prior studies. $^{3536}$

\section{Outcome measures}

We will conduct a series of meta-analyses on multiple health-related outcomes for both stroke survivors and caregivers. As for the primary outcomes of stroke survivors and caregivers, QoL will be measured using generic or condition-specific scales, such as the EQ5D and the StrokeSpecific Quality of Life Scale. Concerning the secondary outcomes of stroke survivors, self-efficacy, coping ability, emotional functioning, self-care behaviour, social participation and ADL will be included. The secondary outcomes of stroke caregivers include self-efficacy, burden, coping ability and emotional functioning.

\section{Statistical analysis}

We will calculate descriptive statistics using SPSS V.21.0, so as to find out the basic characteristics of the included studies, study samples, interventions and others. When the results obtained from the same study are reported through different papers, they will be grouped as companion papers and the results will be reported as a single study. The risk ratio with $95 \%$ CI will be used to express the estimate of the effect for dichotomous outcome. When the same outcome is measured in different ways, the standardised mean difference with $95 \%$ CI will be used to indicate the magnitude of the intervention effect. ${ }^{35}$ The continuous and dichotomous data will be pooled using the inverse variance method and the Mantel-Haenszel method, respectively. ${ }^{36}$ The heterogeneity among the studies will be determined by means of $\chi^{2}$ test. When $\mathrm{p} \geq 0.1$ or $\mathrm{I}^{2} \leq 50 \%$, the fixed effect model will be used to conduct MA. When $\mathrm{p}<0.1$ or $\mathrm{I}^{2}>50 \%$, it is considered that there is statistical heterogeneity among the studies, and the causes of heterogeneity will be analysed. In case of clinical heterogeneity, subgroup analysis or sensitivity analysis will be conducted depending on its source. In case of no significant clinical heterogeneity, MA will be conducted using random effect model. ${ }^{37}$ We will conduct the MA with the assistance of Review Manager V.5.3 software. A narrative summary of the results obtained from individual studies will be made if an MA is not viable.

\section{Validity, reliability and rigour}

We will conduct our study according to the best practices in SR and MA methods. ${ }^{38}{ }^{39}$ The results of SR and MA will be reported according to the PRISMA guidelines. ${ }^{40}$ 
Table 2 The Cochrane Collaboration's tool for assessing risk of bias

\begin{tabular}{|c|c|c|}
\hline Domain & Support for judgement & Review authors' judgement \\
\hline \multicolumn{3}{|l|}{ Selection bias } \\
\hline Random sequence generation & $\begin{array}{l}\text { Describe the method used to generate the } \\
\text { allocation sequence in sufficient detail to } \\
\text { allow an assessment of whether it should } \\
\text { produce comparable groups }\end{array}$ & $\begin{array}{l}\text { Selection bias (biased allocation to } \\
\text { interventions) due to inadequate generation } \\
\text { of a randomised sequence }\end{array}$ \\
\hline Allocation concealment & $\begin{array}{l}\text { Describe the method used to conceal the } \\
\text { allocation sequence in sufficient detail to } \\
\text { determine whether intervention allocations } \\
\text { could have been foreseen in advance of, or } \\
\text { during, enrolment }\end{array}$ & $\begin{array}{l}\text { Selection bias (biased allocation to } \\
\text { interventions) due to inadequate } \\
\text { concealment of allocations prior to } \\
\text { assignment }\end{array}$ \\
\hline \multicolumn{3}{|l|}{ Performance bias } \\
\hline $\begin{array}{l}\text { Blinding of participants and personnel. } \\
\text { Assessments should be made for each } \\
\text { main outcome (or class of outcomes) }\end{array}$ & $\begin{array}{l}\text { Describe all measures used, if any, to } \\
\text { blind study participants and personnel } \\
\text { from knowledge of which intervention } \\
\text { a participant received. Provide any } \\
\text { information relating to whether the intended } \\
\text { blinding was effective }\end{array}$ & $\begin{array}{l}\text { Performance bias due to knowledge of the } \\
\text { allocated interventions by participants and } \\
\text { personnel during the study }\end{array}$ \\
\hline
\end{tabular}

\section{Detection bias}

Blinding of outcome assessment. Assessments should be made for each main outcome (or class of outcomes)
Describe all measures used, if any, to blind outcome assessors from knowledge of which intervention a participant received. Provide any information relating to whether the intended blinding was effective
Detection bias due to knowledge of the allocated interventions by outcome assessors

\section{Attrition bias}

Incomplete outcome data. Assessments should be made for each main outcome (or class of outcomes).

\section{Describe the completeness of outcome} data for each main outcome, including attrition and exclusions from the analysis. State whether attrition and exclusions were reported, the numbers in each intervention group (compared with total randomised participants), reasons for attrition/ exclusions where reported, and any reinclusions in analyses performed by the review authors

\section{Reporting bias}

\section{Selective reporting}

State how the possibility of selective outcome reporting was examined by the review authors, and what was found

\section{Other bias}

Other sources of bias

Attrition bias due to amount, nature or handling of incomplete outcome data

\section{DISCUSSIONS}

In our study, we will compare the efficacy of self-care intervention in stroke survivor-caregiver dyads, thus providing data on the overall effects and identifying the intervention components associated with the most significant improvement of outcomes for patients and/ or caregivers. The results obtained from our study may indicate the direction of further studies on the development of self-care interventions for specific patient groups.
In our study, we will conduct analysis to determine the effect of dyadic self-care interventions on stroke survivor (including QoL, self-efficacy, coping ability, emotional functioning, stroke self-care behaviours, social participation and ADL) and/or caregiver outcomes (including QoL, self-efficacy, burden, coping ability and emotional functioning). This is expected to be the most extensive MA of self-care interventions in stroke survivor-caregiver dyads to date. Through our study, the entry point and components of most effective intervention for the 


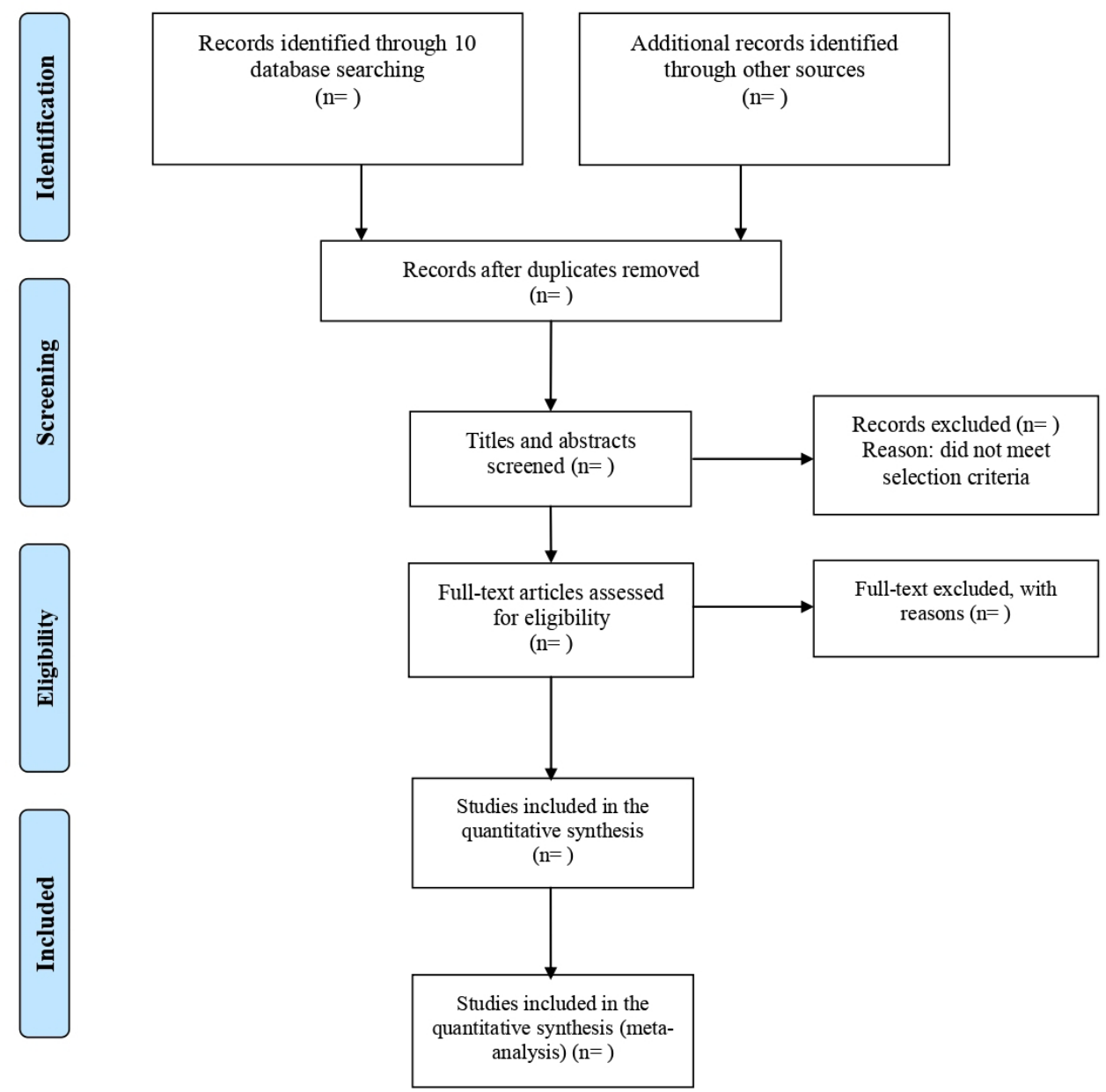

Figure 1 Preferred Reporting Items for Systematic Review and Meta-Analysis 2009 flow diagram.

development of dyadic stroke self-care programme can be identified, which will provide a basis for developing the best method of developing objective measures and interventions with stroke self-care, so as to improve health outcomes for stroke survivors and caregivers. Furthermore, these significant findings will be vital to assisting policymakers and researchers in synthesising a large and complex literature.

\section{Limitations}

Since this is an understudied research area, it is likely that there will be very few studies identified in our review. Also, heterogeneity will arise in MA due to the variation in clinical and methodological characteristics. If it is necessary to amend our protocol considering the potential limitations as mentioned above in this study, the date of each amendment will be presented with a description of the change and corresponding rationale.

\section{PATIENT AND PUBLIC INVOLVEMENT}

There was no time allocated to patient and public involvement, particularly in the context of the current COVID-19 pandemic, so we were unable to involve patients. However, our SR protocol follows a standardised approach as per PRISMA-P guidelines.

\section{ETHICS APPROVAL STATEMENT AND DISSEMINATION}

We will exclude any identifiable patient data. Therefore, ethical approval and participant consent are not required. To conclude the study, we would like to publish our study in a peer-reviewed journal for the findings to be widely disseminated, and our abstract will be presented at suitable national/international conferences.

Acknowledgements Professor Zhiguang Ping is highly appreciated for his assistance with statistical analysis planning.

Contributors All authors have given approval to the final version for publication. The following shows the contributor statement in our study: (1) Study concept and design: WW and BL. (2) Acquisition of data: WW, BL and YM. (3) Drafting of the manuscript: WW, BL, YM and BZ. (4) Critical revision of the manuscript for important intellectual content: all authors. (5) Supervision: ZZ.

Funding This work was supported by the National Natural Science Foundation of China [72174184].

Competing interests None declared.

Patient consent for publication Not applicable.

Provenance and peer review Not commissioned; externally peer reviewed.

Supplemental material This content has been supplied by the author(s). It has not been vetted by BMJ Publishing Group Limited (BMJ) and may not have been peer-reviewed. Any opinions or recommendations discussed are solely those of the author(s) and are not endorsed by BMJ. BMJ disclaims all liability and responsibility arising from any reliance placed on the content. Where the content includes any translated material, BMJ does not warrant the accuracy and reliability of the translations (including but not limited to local regulations, clinical guidelines, 
terminology, drug names and drug dosages), and is not responsible for any error and/or omissions arising from translation and adaptation or otherwise.

Open access This is an open access article distributed in accordance with the Creative Commons Attribution Non Commercial (CC BY-NC 4.0) license, which permits others to distribute, remix, adapt, build upon this work non-commercially, and license their derivative works on different terms, provided the original work is properly cited, appropriate credit is given, any changes made indicated, and the use is non-commercial. See: http://creativecommons.org/licenses/by-nc/4.0/.

ORCID iD

Zhenxiang Zhang http://orcid.org/0000-0003-0326-2834

\section{REFERENCES}

1 Rajsic S, Gothe $\mathrm{H}$, Borba $\mathrm{HH}$, et al. Economic burden of stroke: a systematic review on post-stroke care. Eur J Health Econ 2019;20:107-34.

2 GBD 2016 Stroke Collaborators. Global, regional, and national burden of stroke, 1990-2016: a systematic analysis for the globa burden of disease study 2016. Lancet Neurol 2019;18:439-58.

3 Lin B, Zhang Z, Mei Y, et al. Cumulative risk of stroke recurrence over the last 10 years: a systematic review and meta-analysis. Neurol Sci 2021;42:61-71.

4 Crichton SL, Bray BD, McKevitt C, et al. Patient outcomes up to 15 years after stroke: survival, disability, quality of life, cognition and mental health. J Neurol Neurosurg Psychiatry 2016;87:1091-8.

5 Riegel B, Moser DK, Buck HG, et al. Self-Care for the prevention and management of cardiovascular disease and stroke: a scientific statement for healthcare professionals from the American heart association. J Am Heart Assoc 2017;6. doi:10.1161/ JAHA.117.006997. [Epub ahead of print: 31 Aug 2017].

6 Fryer CE, Luker JA, McDonnell MN, et al. Self management programmes for quality of life in people with stroke. Cochrane Database Syst Rev 2016;8:CD010442.

7 Haley WE, Marino VR, Sheehan OC, et al. Stroke survivor and family caregiver reports of caregiver engagement in stroke care. Rehabil Nurs 2019;44:302-10.

8 Satink T, Josephsson S, Zajec J, et al. Negotiating role management through everyday activities: narratives in action of two stroke survivors and their spouses. Disabil Rehabil 2016;38:2354-64.

9 De Maria M, Vellone E, Ausili D, et al. Self-care of patient and caregiver dyads in multiple chronic conditions: a longitudinal studY (SODALITY) protocol. J Adv Nurs 2019;75:461-71.

10 Pucciarelli G, Lommi M, Magwood GS, et al. Effectiveness of dyadic interventions to improve stroke patient-caregiver dyads' outcomes after discharge: a systematic review and meta-analysis study. Eur $J$ Cardiovasc Nurs 2021;20:14-33.

11 Riegel B, Jaarsma T, Strömberg A. A middle-range theory of self-care of chronic illness. Adv Nurs Sci 2012;35:194-204.

12 Riegel B, Jaarsma T, Lee CS, et al. Integrating symptoms into the middle-range theory of self-care of chronic illness. ANS Adv Nurs Sci 2019;42:206-15

13 Narasimhan M, Allotey P, Hardon A. Self care interventions to advance health and wellbeing: a conceptual framework to inform normative guidance. BMJ 2019;365:1688.

14 Lennon S, McKenna S, Jones F. Self-management programmes for people post stroke: a systematic review. Clin Rehabil 2013;27:867-78.

15 Parke HL, Epiphaniou E, Pearce G, et al. Self-Management support interventions for stroke survivors: a systematic Meta-Review. PLoS One 2015;10:e0131448.

16 Whitehead L. Self-management programmes for quality of life in people with stroke. Int J Nurs Pract 2018;24:e12612.

17 Merriam-Webster. Merriam-Webster. Available: https://www.merriamwebster.com/dictionary/dyad?utm_campaign

18 Lyons KS, Lee CS. The theory of Dyadic illness management. J Fam Nurs 2018;24:8-28.

19 Pucciarelli G, Vellone E, Savini S, et al. Roles of changing physical function and caregiver burden on quality of life in stroke: a longitudinal Dyadic analysis. Stroke 2017;48:733-9.
20 Zhang L, Zhang Z, Mei Y, et al. Dyadic appraisals, dyadic coping, and mental health among couples coping with stroke: a longitudinal study protocol. J Adv Nurs 2020;76:3164-70.

21 Shamseer L, Moher D, Clarke M, et al. Preferred reporting items for systematic review and meta-analysis protocols (PRISMA-P) 2015: elaboration and explanation. BMJ 2015;350:g7647.

22 Stewart LA, Clarke M, Rovers M, et al. Preferred reporting items for systematic review and meta-analyses of individual participant data: the PRISMA-IPD statement. JAMA 2015;313:1657-65.

23 Tielemans NS, Visser-Meily JMA, Schepers VPM, et al. Study protocol of the Restore4Stroke self-management study: a multicenter randomized controlled trial in stroke patients and their partners. Int $J$ Stroke 2014;9:818-23.

24 Tielemans NS, Visser-Meily JMA, Schepers VPM, et al. Effectiveness of the Restore4Stroke self-management intervention "Plan ahead!": A randomized controlled trial in stroke patients and partners. J Rehabil Med 2015;47:901-9.

25 Sureshkumar K, Murthy GVS, Kinra S, et al. Development and evaluation of a Smartphone-enabled, caregiver-supported educational intervention for management of physical disabilities following stroke in India: protocol for a formative research study. BMJ Innov 2015;1:117-26.

26 Chau JPC, Lo SHS, Lee VWY, et al. Effectiveness and costeffectiveness of a virtual multidisciplinary stroke care clinic for community-dwelling stroke survivors and caregivers: a randomised controlled trial protocol. BMJ Open 2019;9:e026500.

27 van Mastrigt GAPG, van Eeden M, van Heugten CM, et al. A trial-based economic evaluation of the Restore4Stroke selfmanagement intervention compared to an education-based intervention for stroke patients and their partners. BMC Health Serv Res 2020;20:294.

28 Kamal A, Khoja A, Usmani B, et al. Effect of 5-Minute movies shown via a mobile phone APP on risk factors and mortality after stroke in a low- to middle-income country: randomized controlled trial for the stroke caregiver dyad education intervention (Movies4Stroke). JMIR Mhealth Uhealth 2020;8:e12113.

29 Helmer D, Savoie I, Green C, et al. Evidence-based practice: extending the search to find material for the systematic review. Bull Med Libr Assoc 2001;89:346-52.

30 McAuley L, Pham B, Tugwell P, et al. Does the inclusion of grey literature influence estimates of intervention effectiveness reported in meta-analyses? Lancet 2000;356:1228-31.

31 Higgins JPT, Altman DG, Gøtzsche PC, et al. The Cochrane collaboration's tool for assessing risk of bias in randomised trials. BMJ 2011;343:d5928.

32 Hróbjartsson A, Boutron I, Turner L. Assessing risk of bias in randomised clinical trials included in Cochrane reviews: the why is easy, the how is a challenge. Cochrane Database Syst Rev 2013;4:ED000058.

33 Ruppar TM, Cooper PS, Johnson ED, et al. Self-care interventions for adults with heart failure: a systematic review and meta-analysis protocol. J Adv Nurs 2019;75:676-82.

$34 \mathrm{McHugh}$ ML. Interrater reliability: the kappa statistic. Biochem Med 2012;22:276-82.

35 Wan X, Wang W, Liu J, et al. Estimating the sample mean and standard deviation from the sample size, median, range and/or interquartile range. BMC Med Res Methodol 2014;14:135.

36 Cumpston M, Li T, Page MJ, et al. Updated guidance for trusted systematic reviews: a new edition of the Cochrane Handbook for systematic reviews of interventions. Cochrane Database Syst Rev 2019;10:ED000142.

37 Zhai J, Mu W, Si J, et al. Acupuncture for constipation in patients with stroke: protocol of a systematic review and meta-analysis. BMJ Open 2018;8:e020400.

38 Cheung MW-L, Vijayakumar R. A guide to conducting a metaanalysis. Neuropsychol Rev 2016;26:121-8.

39 Muka T, Glisic M, Milic J, et al. A 24-step guide on how to design, conduct, and successfully publish a systematic review and metaanalysis in medical research. Eur J Epidemiol 2020;35:49-60.

40 Moher D, Liberati A, Tetzlaff J, et al. Preferred reporting items for systematic reviews and meta-analyses: the PRISMA statement. J Clin Epidemiol 2009;62:1006-12. 